\title{
OVI in the local interstellar medium: FUSE observations towards B-stars within 200 pc
}

\author{
B. Y. Welsh ${ }^{1}$ and R. Lallement ${ }^{2}$ \\ 1 Space Sciences Laboratory, University of California, 7 Gauss Way, Berkeley, CA 94720, USA \\ e-mail: bwelsh@ssl. berkeley .edu \\ 2 Service d'Aéronomie du CNRS, 91371 Verrières-le-Buisson, France \\ Received 19 May 2008 / Accepted 4 September 2008 \\ ABSTRACT

\begin{abstract}
Aims. We present far ultraviolet (FUV: 990-1082 Å) spectroscopic observations of 17 early-type stars located within 200 pc of the Sun using the NASA FUSE satellite in order to search for interstellar absorption from the important high ion of OVI (1032 ̊̊).

Methods. These spectral data were fit with a local stellar continuum such that measurement of, or upper limits to, the equivalent width of the OVI absorption line could be made. The resultant line profiles were then fit with an absorption model to determine the OVI column density along each sight-line.

Results. OVI was detected towards 5 of the 17 targets, all towards stars with distances $>80 \mathrm{pc}$. The average volumic density, $n_{\mathrm{OVI}}$, along these 5 sight-lines was found to be $5.2 \times 10^{-8} \mathrm{~cm}^{-3}$. This value, in agreement with measurements of OVI in the local interstellar medium (ISM) by other authors, is measurably higher than that typically found throughout the general ISM. The location of these 5 stars places them just within, or just at, the neutral boundary to the Local Cavity. If this cavity contains a hot million degree K gas, then it seems likely that the presently observed OVI absorption is formed in conductive interfaces within a transition layer between hot and cooler gas. We provide tentative evidence that the spatial distribution of OVI absorbers and emitters exhibits a loose correlation with that of the million degree K soft X-ray background (SXRB) emission at galactic latitudes of $+30^{\circ}<b<-30^{\circ}$. In such a scenario very little SXRB emission is required in the galactic plane, in agreement with current theoretical models that invoke solar wind charge exchange as the origin for the majority of this background signal. Alternately, if the Local Cavity is an old SNR of age $\sim 3$ million yr, it is possible that the central regions may no longer contain soft X-ray emitting gas, with appreciable amounts of OVI ions lying within warm $\left(T \sim 10^{6} \mathrm{~K}\right)$ gas at the periphery of the bubble cavity.
\end{abstract}

Key words. Galaxy: solar neighborhood - ISM: atoms - ultraviolet: ISM

\section{Introduction}

The interstellar region surrounding our Sun to $\sim 100 \mathrm{pc}$ in all directions, hereafter termed the "Local Cavity" (LC), is one of unusually low neutral interstellar gas density $\left(n_{\mathrm{HI}}<0.1 \mathrm{~cm}^{-3}\right)$. Its approximate dimensions and associated neutral gas boundary have been mapped using $\mathrm{NaI}$ absorption measurements towards $\sim 1050$ nearby stars by Lallement et al. (2003). Since the LC is largely free of large condensations of cold and dense neutral gas (although it does contain many rarefied partially ionized cloudlets, often referred to as the local fluff (Lallement et al. 1986), it has been natural to assume that it is filled with a hot and low density plasma. Historically, support for this latter notion was based mainly on (a) theoretical models of the interstellar medium (ISM) that required the presence of a pervasive hot gasphase component (Cox \& Smith 1974; McKee \& Ostriker 1977); and (b) observations of the soft X-ray $(0.25 \mathrm{keV})$ background radiation, whose emission properties have been associated with a $T \sim 10^{6} \mathrm{~K}$ gas located within the solar interstellar neighborhood (Williamson et al. 1977; Sanders et al. 1977). Further support for the presence of local gas with temperatures in the $30000 \mathrm{~K}$ range came from the apparent detection of the highly ionized interstellar absorption line of OVI $\lambda 1032 \AA$ towards B-stars within $100 \mathrm{pc}$ using the Copernicus satellite (Jenkins 1978). These observations were followed by the HST detections of interstellar SiIV $\lambda 1394 \AA$ and CIV $\lambda 1550 \AA$ absorption (with a lower gas temperature of $\leq 10^{5} \mathrm{~K}$ ) along sight-lines towards several local hot white dwarfs (Holberg et al. 1998). From all these (and other) observational data a compelling picture of a rarefied, lowdensity interstellar bubble containing highly ionized million degree $\mathrm{K}$ gas that surrounds the solar neighborhood has therefore emerged.

However over the past decade some observational evidence, that seems in stark contradiction to the aforementioned picture of the local ISM, has emerged. Firstly, both the NASA EUVE and CHIPS missions have failed to detect the EUV line emission which was expected from a diffuse hot LC plasma emitting with a temperature of $\sim 10^{6} \mathrm{~K}$ (Jelinsky et al. 1995; Hurwitz et al. 2005). The non-detection of this EUV background emission signal has been interpreted as (a) the local hot gas having a very low iron abundance and a far lower gas density than previously thought; or (b) being due to the absence of million degree hot gas within the LC. This latter interpretation would at first seem untenable, given the existence of the soft X-ray background (SXRB) signal whose $\mathrm{Be}$ and $B$-band ratios suggest its formation within a low-density million degree K plasma located within 100 pc (Bloch et al. 1986). The more recent ROSAT satellite measurements of the $0.25 \mathrm{keV}$ SXRB signal have been interpreted as comprising of both an unabsorbed (LC) foreground component and a more distant component that originates in either the galactic halo or from an extragalactic background source (Snowden et al. 1998). This scenario seemed to fit most models 
of the LC, except that the value derived for the pressure of the hot X-ray emitting gas was far above that measured in colder gas clouds embedded within the local ISM (Lallement 1998; Jenkins 2002). Such a pressure imbalance would result in a shock with a velocity of $\sim 20 \mathrm{~km} \mathrm{~s}^{-1}$ being driven into the local cloud, which is contrary to all observations. In addition, the actual spectral signatures of the SXRB emission line spectrum could not be characterized by either collisional equilibrium models nor with nonequilibrium ionization models of an emitting hot gas (Sanders et al. 2001).

It has only recently been realized that a significant fraction of the SXRB signal can arise in the interplanetary medium of the heliosphere via charge exchange between solar wind heavy ions and interstellar neutral ions (Robertson \& Cravens 2003; Lallement 2004). Furthermore, attempts to isolate a foreground soft X-ray signal (originating within the LC) from that observed towards more distant sources by cloud shadowing techniques have led to inconclusive results (Smith et al. 2007; Henley \& Shelton 2007). Recently, Koutroumpa et al. (2007) have shown that all $0.56 \mathrm{keV}$ OVII and $0.65 \mathrm{keV}$ OVIII emission detected as a shadow foreground are of a heliospheric origin, demonstrating that such observations cannot be used to prove the existence of a local hot gas. Thus, although there must be some contribution to the SXRB signal at high galactic latitudes (presumably from a hot and ionized galactic halo), the evidence for widespread million degree gas located within $\sim 100 \mathrm{pc}$ of the Sun (particularly in the galactic plane) is becoming less compelling.

However, UV absorption observations towards stars within $100 \mathrm{pc}$ have provided strong support for the presence of a hot and highly ionized LC plasma (Jenkins 1978; Holberg et al. 1998). According to several theoretical models, evaporative conductive interfaces between ambient hot $\left(10^{6} \mathrm{~K}\right)$ and embedded cold/warm (local) clouds should produce semi-hot $\left(\sim 10^{5} \mathrm{~K}\right)$ gas that contains highly ionized ions such as SiIV, CIV, NV and OVI (Slavin \& Frisch 2002; Indebetouw \& Shull 2004). The majority of these UV absorption observations have used (faint) hot white dwarf stars as background probes of these local interstellar sightlines, since nearby early-type stars are normally intrinsically too bright and typically cause detector saturation. Hot white dwarfs were also thought to have the added bonus of possessing a simple UV continuum against which even weak levels of line absorption could be potentially detected. Unfortunately, the intrinsic complexity of EUV and FUV hot white dwarf spectra (due to the presence of heavy elements in their atmospheres) has now been revealed, such that many of these weak UV absorption features previously attributed to high ion absorption from CIV, SiIV and NV in the hot LC gas may not be of an interstellar origin (Bannister et al. 2003; Holberg et al. 1997). Several recent studies have also revealed the preponderance of circumstellar metal-rich debris disks around many nearby (hydrogen and helium rich) cool white dwarfs, which can potentially provide a source for accretion of metals and dust into the underlying stellar atmospheres (Zuckerman et al. 2003; Koester \& Wilken 2006; Kilic \& Redfield 2007).

The two recent surveys of local OVI absorption using the NASA FUSE satellite by Savage \& Lehner (2006) and Oegerle et al. (2005) have made meaningful observations along 39 sightlines. These surveys have revealed only six $(>3 \sigma)$ interstellar OVI detections towards the 13 sight-lines with distances $<85 \mathrm{pc}$ of the Sun (i.e. $\sim 45 \%$ detection rate within the LC). The overall spatial distribution of local interstellar OVI absorption is described as being "patchy", which has been interpreted as this being due to the presence of tangled or tangential magnetic fields which can quench the thermally conductive interfaces of cloud surfaces on which the OVI is though to form (Cox \& Helenius 2003). A field strength of $8 \mu \mathrm{G}$ has recently been determined for the boundary wall to the LC, thus supporting the idea of an internally high magnetic pressure (Andersson \& Potter 2006). We note that Barstow et al. (2008) have recently carried out a reanalysis of the FUSE absorption data recorded towards $\sim 100$ hot white dwarf stars within $200 \mathrm{pc}$, using a detailed comparison of the radial velocities of the interstellar and (when present) stellar absorption lines. In many cases, they find the velocity differences between the interstellar and photospheric components are below the resolution of the FUSE spectrographs. However, they claim that in the majority of stars where an O VI absorption feature is clearly detected, the material is more consistent with its formation in a circumstellar or photospheric environment rather than that of an interstellar origin. They find a small number of linesof-sight where the OVI absorption is clearly of an interstellar origin, with most of these directions being towards stars located close to, or just beyond, the neutral boundary of the LC.

In addition, the new Hipparcos distance estimates (ESA 1997) to many of the B-type stars originally observed by Copernicus (Jenkins 1978) now result in only one tentative detection of interstellar OVI for a sight-line contained within the neutral boundaries of the LC, i.e. that of $\alpha$ Eri $(d=44 \mathrm{pc})$. However, we note that this Copernicus observation has recently been re-appraised and assigned a large measurement error that is almost equal to the previously reported OVI column density value (Bowen et al. 2008). Thus, it is clear from all of these recent findings that many questions still remain to be answered as to the actual physical state of the LC interstellar gas within $100 \mathrm{pc}$ and how this phase compares and relates to gas in the general diffuse ISM. In order to investigate this issue further, we have extracted FUSE spectra of 17 early (B and A-type) stars located within $200 \mathrm{pc}$ of the Sun from the Multi-Mission Archive at the Space Telescope Science Institute (MAST) in order to determine the properties of interstellar OVI absorption along these nearby sight-lines. Such data have the advantages that the B-star distances are well known, the spectra are not contaminated by photospheric features and several of the sight-lines have previously determined interstellar structure recorded with high resolution ground-based visible absorption spectra of the $\mathrm{NaI}$ or CaII lines.

\section{Observations and data reduction}

Potential B-type (and A0V-type) target stars were selected from observations recorded with the NASA FUSE satellite (Moos et al. 2000, 2002) prior to its end-of-mission in mid-2007. Only stars with parallax distances $<200 \mathrm{pc}$ and spectral data recorded in the 980-1080 $\AA$ region were selected for further investigation. These far UV observations were recorded as either part of the (curtailed) FUSE Legacy program, F329, or as publicly accessible data files extracted from the Multi-Mission Archive at the Space Telescope Science Institute (MAST). We have only used data associated with either the LiF1a or LiF2b spectral channels recorded using either the FUSE LWRS or MDRS apertures (for further details of the FUSE instrument and observational strategies see Sahnow et al. 2000). The majority of these data have been processed using version 3.1 or later of the CALFUSE science data reduction pipeline (Dixon et al. 2007). This science data pipeline corrects for geometric image distortions, background subtraction, image thermal drifts, detector deadtime, wavelength and flux calibration.

Our data search resulted in finding useable far UV spectra for 16 B-type and one A0V-type stars located within $200 \mathrm{pc}$ of the 
Table 1. Stellar target information.

\begin{tabular}{cccccccc}
\hline \hline Star & $\begin{array}{c}(l, b) \\
(\mathrm{deg})\end{array}$ & Sp & $\begin{array}{c}V_{\text {ROT }} \\
\left(\mathrm{km} \mathrm{s}^{-1}\right)\end{array}$ & $\begin{array}{c}\text { Distance } \\
(\mathrm{pc})\end{array}$ & $\begin{array}{c}V_{\text {LISM }} \\
\left(\mathrm{km} \mathrm{s}^{-1}\right)\end{array}$ & Interstellar reference & FUSE Data Set I.D. \\
\hline HD 560 & $(106.9,-50.4)$ & B9V & 249 & $100 \pm 9$ & -20.8 & FUSE* $^{*}$ & P119060800 \\
HD 20863 & $(147.2,-07.0)$ & B9V & 200 & $169 \pm 20$ & +4.0 & FUSE* $^{*}$ & Z9011002000 \\
HD 26670 & $(144.8,+08.0)$ & B5V & 270 & $125 \pm 10$ & +9.3 & FUSE* $^{*}$ & F3290902000 \\
HD 74604 & $(148.2,+36.2)$ & B8V & 150 & $158 \pm 18$ & -11.5 & FUSE* & Z9012401000 \\
HD 75311 & $(273.9,-08.4)$ & B3V & $>300$ & $168 \pm 12$ & +14.0 & NaI, CaII** & F3292901000 \\
HD 75416 & $(292.4,-21.7)$ & B8V & N/A & $97 \pm 5$ & +11.3 & FUSE* & G0480301000 \\
HD 90972 & $(270.1,+23.1)$ & B9.5V & 30 & $147 \pm 15$ & +12.0 & NaI, CaII** & Q1140201000 \\
HD 110020 & $(301.8,-03.7)$ & B8V & N/A & $108 \pm 8$ & +5.0 & NaI, CaII** & U1084501000 \\
HD 124367 & $(314.1,+04.0)$ & B4V & 301 & $149 \pm 12$ & +1.0 & FUSE* & F3293501000 \\
HD 139006 & $(41.9,+53.8)$ & A0V & 138 & $23 \pm 1$ & -17.0 & FUSE* & B0910301000 \\
HD 148594 & $(350.9,+13.9)$ & B8V & 300 & $167 \pm 25$ & -8.0 & Crawford (2000) & P2310101000 \\
HD 149630 & $(66.9,+42.7)$ & B9V & 303 & $93 \pm 6$ & -10.0 & FUSE* & B0910101000 \\
HD 149730 & $(330.4,-06.8)$ & B9IV & N/A & $80 \pm 11$ & +4.5 & FUSE* & Z902012000 \\
HD 150100 & $(80.9,+41.5)$ & B9.5V & 70 & $123 \pm 10$ & +3.8 & FUSE* & C0500201000 \\
HD 176638 & $(354.9,-19.8)$ & B9.5V & N/A & $56 \pm 3$ & -30.0 & CaII* & B0910201000 \\
HD 188665 & $(90.6,+14.9)$ & B5V & 105 & $196 \pm 20$ & -8.0 & NaI, CaII** & F3290701000 \\
HD 201908 & $(112.4,+20.2)$ & B8V & N/A & $126 \pm 8$ & -19.0 & FUSE* & Z9017401000 \\
\hline
\end{tabular}

$*$ = Average velocity of the interstellar OI and CII lines measured from the FUSE data; ** = average velocity of the interstellar NaI and/or CaII lines, Welsh \& Lallement (2008).

Sun. A list of these 17 stellar targets, together with their galactic co-ordinates, spectral type, rotational velocity (when known) and Hipparcos distance are shown in Table 1. In Col. 6 we also list the value of the interstellar velocity $\left(V_{\text {LISM }}\right)$ of the NaI-D and/or CaII-K line (in the heliocentric frame) for each sightline, when available from the relevent references given in Col. 7 . When such velocity values were not available, in Col. 6 we list the average velocity of the CII $\lambda 1036 \AA$ and OI $\lambda 1039 \AA$ interstellar lines as measured directly from the FUSE data. Finally, we also list the FUSE identifier for the data sets used in our subsequent analysis of each target sight-line.

Since these target stars are bright in the UV, many of the FUSE observations were recorded using many short exposures with the data having been recorded in the detector histogram mode. In such cases individual exposure files were co-aligned and then added in order to improve the final $\mathrm{S} / \mathrm{N}$ of the stellar spectrum. Since the FUSE wavelength scale, as calculated with the science pipeline, is known to produce variable shifts of $\pm 20 \mathrm{~km}^{-1}$ across the spectral region, each spectrum was coaligned by eye using the (narrow) OI $\lambda 1039.23 \AA$ interstellar absorption line as a primary fiducial. The typical accuracy of this alignment procedure is $\sim 0.013 \AA$ (i.e. $\pm 4 \mathrm{~km} \mathrm{~s}^{-1}$ ). The coadded exposure data were subsequently binned into $\sim 0.026 \AA$ wavelength samples, such that the final spectrum consisted of 3 sampled points per spectral resolution element. The resolving power of all these data is $20000\left(15 \mathrm{~km} \mathrm{~s}^{-1}\right)$, with all spectra being of a $S / N$ ratio $\geq 7: 1$ at a wavelength of $1032 \AA$.

In order to improve on the final wavelength accuracy of the spectra, when possible, use was made of the more accurate heliocentric velocities found from high resolution observations of the interstellar CaII-K and/or NaI-D lines towards these stars. When such data were available (see Table 1) we have assumed that the interstellar OI $\lambda 1039.23 \AA$ line is formed at the same (heliocentric) velocity as that of the main absorption component of these visible lines and the FUSE wavelength scale was then shifted accordingly to obtain parity. When no visible absorption data were available for a particular sight-line, we used the wavelength scale derived directly from the FUSE pipeline. We note that Savage \& Lehner (2006) have used a similar wavelength scaling technique for their OVI data and found that the relative velocity calibration between the interstellar OVI, OI and CII $\lambda 1036.34 \AA$ lines is accurate to $\sim 5 \mathrm{~km} \mathrm{~s}^{-1}$. The reader should note that the main conclusions of this paper do not dependent on arguments based upon accurately measured interstellar velocities and we present such values purely for informational/comparison purposes.

\subsection{Measurement of equivalent widths, upper limits and column densities}

The main focus of our present interstellar analysis is study of the important OVI $\lambda 1031.93 \AA$ absorption line, but we have also extracted spectral information from the nearby interstellar lines of OI $\lambda 1039.23 \AA$ and CII $\lambda 1036.34 \AA$ mainly for the purpose of absorption and velocity comparison. No measurements were made for the intrinsically weaker OVI $\lambda 1037.616 \AA$ absorption line, whose strength for these data was less than the measured continuum noise value.

Our initial step in the data reduction process was to fit a local stellar continuum extending $\pm 200 \mathrm{~km} \mathrm{~s}^{-1}$ either side of the observed wavelength of each of the interstellar lines of interest. Typically this procedure required a third (or higher) order polynomial in order to produce a residual line intensity profile of the absorption line, such that a total line equivalent width $\left(W_{\lambda}\right)$ could be subsequently determined. We note that the continuum placement was relatively straight-forward since none of our spectra reveal absorption contamination from the nearby molecular $\mathrm{H}_{2}$ lines at $1031.10 \AA$ and $1032.36 \AA$, presumably due to the fact the short path lengths sampled by these sight-lines do not pass through any very dense and cold regions within the local ISM. Also, most of these targets are fast rotators, such that contamination by any narrow stellar photospheric features near the OVI wavelength is not problematic. We note that B stars often possess strong P-Cygni profiles due to the winds driven by massive stars and thus the interstellar OVI lines lie deep within the absorption trough of the stellar line making their detection difficult for the short interstellar sight-lines presently sampled (Bowen et al. 2008). In addition, several authors have shown that variable absorption features in stellar winds can impact interstellar absorption measurements 
Table 2. OVI column density summary.

\begin{tabular}{|c|c|c|c|c|c|c|c|c|c|c|}
\hline \multirow[b]{2}{*}{ star } & \multicolumn{5}{|c|}{ OVI(1032 A) } & \multicolumn{3}{|c|}{ OI(1039 ̊̊) } & \multicolumn{2}{|l|}{ CII(1036 ̊) } \\
\hline & SNR & $\begin{array}{c}W_{\lambda}(\mathrm{OVI}) \\
(\mathrm{m} \AA) \\
\end{array}$ & $\begin{array}{c}V_{\odot} \\
\left(\mathrm{km} \mathrm{s}^{-1}\right) \\
\end{array}$ & $\begin{array}{c}b \\
\left(\mathrm{~km} \mathrm{~s}^{-1}\right) \\
\end{array}$ & $\begin{array}{c}\log N(\mathrm{OVI}) \\
\left(\mathrm{cm}^{-2}\right)\end{array}$ & $\begin{array}{c}n_{\mathrm{OVI}} \\
\left(\mathrm{cm}^{-3}\right) \\
\end{array}$ & $\begin{array}{c}W_{\lambda}(\mathrm{OI}) \\
(\mathrm{m} \AA) \\
\end{array}$ & $\begin{array}{c}V_{\odot} \\
\left(\mathrm{km} \mathrm{s}^{-1}\right) \\
\end{array}$ & $\begin{array}{c}W_{\lambda}(\mathrm{CII}) \\
(\mathrm{m} \AA) \\
\end{array}$ & $\begin{array}{c}V_{\odot} \\
\left(\mathrm{km} \mathrm{s}^{-1}\right) \\
\end{array}$ \\
\hline HD 560 & $9: 1$ & $<13.7$ & - & - & $<13.04$ & $<3.5 \times 10^{-8}$ & $76.5 \pm 10$ & -21.5 & $90.5 \pm 13$ & -20.0 \\
\hline HD 20863 & $9: 1$ & $30.0 \pm 9.5$ & +6.0 & $13.5 \pm 4$ & $13.48 \pm 0.09$ & $5.8 \times 10^{-8}$ & $110.0 \pm 30$ & +4.0 & $98.5 \pm 20$ & +4.0 \\
\hline HD 26670 & $7: 1$ & $<17.5$ & - & - & $<13.15$ & $<3.7 \times 10^{-8}$ & $74.5 \pm 8$ & +6.0 & $107 \pm 35$ & +12.5 \\
\hline HD 74604 & $17: 1$ & $11.5 \pm 4.0$ & -4.4 & $13.6 \pm 5$ & $12.98 \pm 0.15$ & $2.0 \times 10^{-8}$ & $60.0 \pm 10$ & -15.0 & $85 \pm 10$ & -9.0 \\
\hline HD 75311 & $14: 1$ & $<10.5$ & - & - & $<12.93$ & $<1.6 \times 10^{-8}$ & $90.0 \pm 9$ & +13.1 & $115 \pm 15$ & +14.5 \\
\hline HD 75416 & $10: 1$ & $<12.7$ & - & - & $<13.00$ & $<3.3 \times 10^{-8}$ & $78.0 \pm 9$ & +11.0 & $115 \pm 20$ & +11.5 \\
\hline HD 90972 & $10: 1$ & $<10.6$ & - & - & $<12.92$ & $<1.8 \times 10^{-8}$ & $55.0 \pm 8$ & +12.0 & $86 \pm 14$ & +4.5 \\
\hline HD 110020 & $7: 1$ & $<16.8$ & - & - & $<13.13$ & $<4.0 \times 10^{-8}$ & $65.0 \pm 8$ & +4.5 & $89 \pm 17$ & +2.0 \\
\hline HD 124367 & $18: 1$ & $27.5 \pm 8.5$ & -5.8 & $14.6 \pm 4$ & $13.39 \pm 0.13$ & $5.3 \times 10^{-8}$ & $105 \pm 10$ & -2.0 & $175 \pm 27$ & +4.0 \\
\hline HD 139006 & $10: 1$ & $<15.5$ & - & - & $<13.09$ & $<1.7 \times 10^{-7}$ & $15.5 \pm 5$ & -18.0 & $61 \pm 9$ & -16.0 \\
\hline HD 148594 & $7: 1$ & $<14.0$ & - & - & $<13.05$ & $<2.2 \times 10^{-8}$ & $145 \pm 15$ & -7.5 & $\mathrm{~N} / \mathrm{A}^{*}$ & - \\
\hline HD 149630 & $14: 1$ & $22.0 \pm 7.0$ & +16.9 & $19.1 \pm 6$ & $13.32 \pm 0.23$ & $7.3 \times 10^{-8}$ & $45.0 \pm 8$ & -11.0 & $150 \pm 11$ & -12.0 \\
\hline HD 149730 & $13: 1$ & $17.5 \pm 6.0$ & -11.2 & $20.8 \pm 6$ & $13.16 \pm 0.13$ & $5.8 \times 10^{-8}$ & $115.0 \pm 12$ & 0.0 & $180 \pm 20$ & +9.0 \\
\hline HD 150100 & $12: 1$ & $<8.5$ & - & - & $<12.85$ & $<1.9 \times 10^{-8}$ & $80.2 \pm 11$ & $\begin{array}{l}0.0 \\
+2.0\end{array}$ & $121 \pm 11$ & +5.5 \\
\hline HD 176638 & $10: 1$ & $<13.3$ & - & - & $<13.03$ & $<6.2 \times 10^{-8}$ & $53.0 \pm 8$ & -31.0 & $84 \pm 12$ & -26.0 \\
\hline HD 188665 & $13: 1$ & $<7.6$ & - & - & $<12.83$ & $<1.1 \times 10^{-8}$ & $101.6 \pm 10$ & -8.5 & $106 \pm 24$ & -8.6 \\
\hline HD 201908 & $7: 1$ & $<13.5$ & - & - & $<13.03$ & $<2.8 \times 10^{-8}$ & $93.0 \pm 8$ & -19.5 & $112 \pm 13$ & -18.5 \\
\hline
\end{tabular}

* = Saturated line blended with CII*1037 ̊.

(Bjorkman et al. 1994). Unfortunately, none of our stars were observed at multiple epochs and thus we are presently unable to assess such an effect on our data.

The $\mathrm{S} / \mathrm{N}$ ratios for these continuum fits are given in Col. 2 of Table 2, and the values of $W_{\lambda}$, along with their measurement errors are listed for the OVI, OI and CII lines. The error on $W_{\lambda}$ is derived from a function of both the $\mathrm{S} / \mathrm{N}$ ratio of the local continuum and the number of data points contained within each of the absorption line-profiles (Welsh et al. 1990).

For the (five) instances in which OVI absorption was detected with a significance $>2.5-\sigma$, their interstellar line profiles were then fit with a single absorption component using the linefitting procedure described in Sfeir et al. (1999). This procedure generates a best-fit theoretical absorption profile that is characterized by 3 parameters: (i) a doppler velocity dispersion, $b$-value; (ii) an interstellar cloud (heliocentric) velocity, $V$; and (iii) a cloud component column density, $N$. When convolved with the FUSE instrument resolution we obtain the best-fit absorption profiles shown in Figs. 1 and 2 for the five OVI detections. The corresponding best-fit values of $b, V$ and $N$ for these OVI absorption-line detections are listed in Table 2, together with the error on the derived best-fit $b$-value. The error on the placement of the absorption component velocity was found to be $\sim \pm 4 \mathrm{~km} \mathrm{~s}^{-1}$ for all the fits, which is comparable to the estimated $\pm 5 \mathrm{~km} \mathrm{~s}^{-1}$ absolute accuracy of the FUSE wavelength scale. For comparison purposes, in Figs. 1 and 2 we also plot the normalized CII and OI interstellar line profiles observed towards the 5 stars in which OVI was detected.

The observed level of interstellar OVI absorption for all of these local sight-lines is generally quite weak (i.e. $W_{\lambda}<30 \mathrm{~m} \AA$ ), and since these profiles are superposed on a typically low $\mathrm{S} / \mathrm{N}$ continuum level for these particular FUSE observations, obtaining an accurate assessment of an upper limit for any absorption feature near to the expected wavelength of OVI is therefore critically important for any subsequent scientific conclusions arising from these data. In addition, it has now been realized that the limited $\mathrm{S} / \mathrm{N}$ of the flat field applied by the FUSE data pipeline
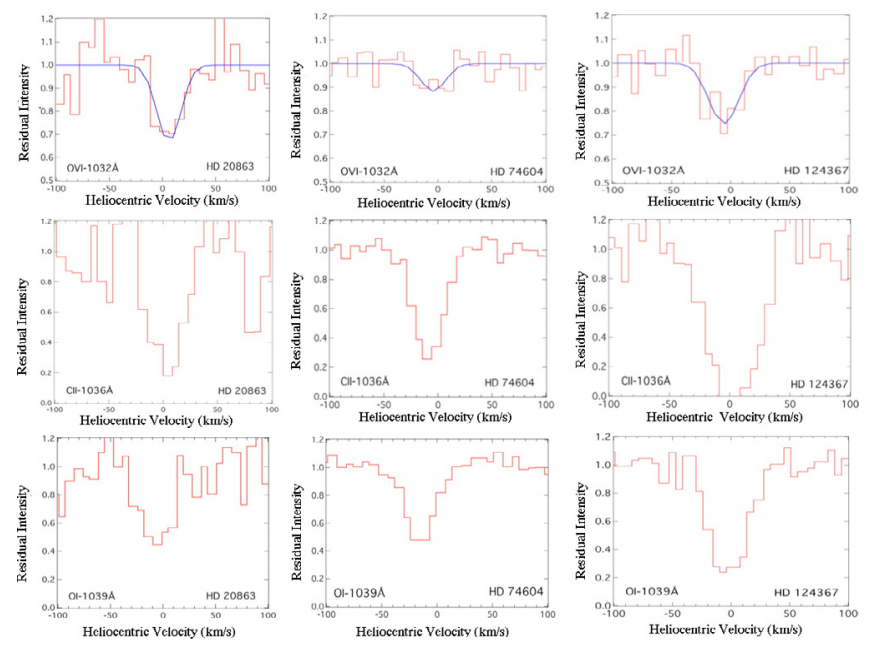

Fig. 1. Normalized profiles vs. heliocentric velocity of the interstellar OVI detections for HD 20863, HD 74604 and HD 124367. The thick bold line is the best-fit model to the observed OVI data points. Also shown on the same velocity scale are the normalized profiles of the CII (1035 ̊) and OI (1039 ̊) interstellar lines.

does not totally account for the removal of weak and narrow absorption features caused by the so-called hex-boundaries of the detector micro-channel plates. Therefore in order to assess if a weak absorption "feature" that may appear in the spectra near the expected wavelength of an interstellar OVI line is of true astrophysical origin, we have carried out the following data reduction procedures in order to determine the statistical significance of such absorptions with respect to the actual $\mathrm{S} / \mathrm{N}$ of the observed data points.

We start by first fitting the entire stellar continuum extending $\pm 200 \mathrm{~km} \mathrm{~s}^{-1}$ either side of the expected central rest wavelength $\left(\lambda_{\mathrm{c}}\right)$ of the interstellar OVI line with a third order polynomial or greater, to produce a spectrum of residual intensities that is quasi-symmetric about unity. We then constrain our search for 

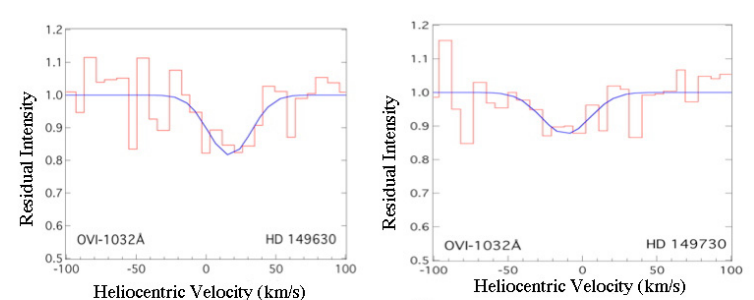

Heliocentric Velocity $(\mathrm{km} / \mathrm{s})$
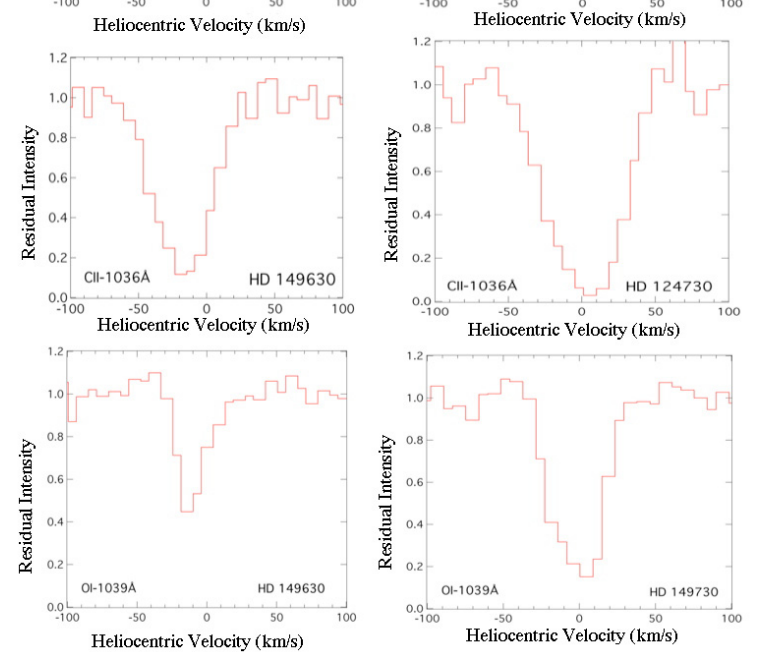

Fig. 2. Normalized profiles vs. heliocentric velocity of the interstellar OVI detections for HD 149630 and HD 149730. The thick bold line is the best-fit model to the observed OVI data points. Also shown on the same velocity scale are the normalized profiles of the CII (1035 $\AA$ ) and OI (1039 ̊) interstellar lines.

potential interstellar absorption features in these data to within $\pm 100 \mathrm{~km} \mathrm{~s}^{-1}$ of $\lambda_{\mathrm{c}}$. This range encompasses interstellar absorption velocities that are typically found for gas clouds within the local ISM. We then start a search for absorption features in the spectra that are consistent both with the expected line-width of the OVI interstellar line (due to the convolution of the expected doppler-width of the line and the instrumental resolving power), and are also statistically significant with respect to the local S/N of the data points. For each data point with velocity, $V(i)$, between -100 and $+100 \mathrm{~km} \mathrm{~s}^{-1}$ of $\lambda_{\mathrm{c}}$ we construct a theoretical best-fit gaussian profile using $V(i)$ as the fixed center wavelength in order to derive a column density, $N(i)$, of the level of absorption (or emission) that is consistent with the observed residual intensity data points that are contained within the gaussian. This procedure is repeated for every point over the velocity range of interest such that a histogram of values of $N(i)$ for each gaussian is obtained. This distribution of absorption and emission line strengths is approximately symmetric about zero column density with a width that corresponds to the errors associated with measurement from the data. The width of this column density value distribution is $\sigma$, and we report values of $2-\sigma$ as a conservative upper limit to the column density, $N$, of any real interstellar absorption feature in these data. For the OVI line we adopted a doppler-width (i.e. $b$-value) of $17 \mathrm{~km} \mathrm{~s}^{-1}$, which is a typical intrinsic line width for a gas near its peak abundance at a temperature of $2.85 \times 10^{5} \mathrm{~K}$.

In Table 2 we also report the corresponding 2- $\sigma$ upper limits to the equivalent widths of these features by assuming a constant of proportionality with the derived OVI column density upper limit value, $N(\mathrm{OVI})$. In Fig. 3 we show four examples of these instances in which we were unable to detect a significant level of OVI absorption. The 2- $\sigma$ values of OVI equivalent width for these four examples range from $7.6 \mathrm{~m} \AA$ to $17.5 \mathrm{~m} \AA$.
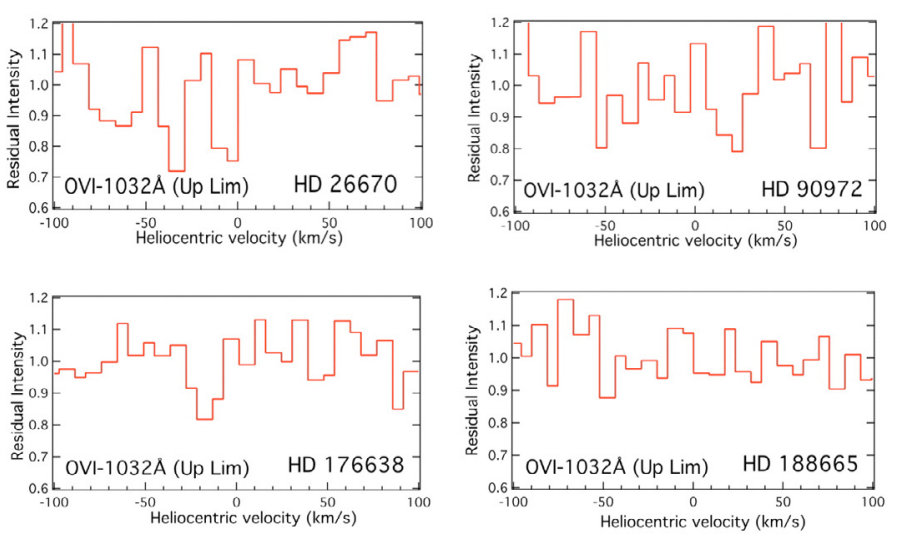

Fig. 3. Examples of sight-lines with only upper limits to OVI interstellar

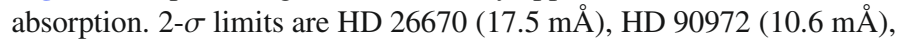

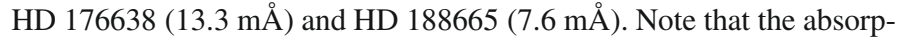
tion feature at $-20 \mathrm{~km} \mathrm{~s}^{-1}$ seen towards HD 176638 is too narrow to be consistent with that expected from an OVI absorption line.

\section{Discussion}

\subsection{OVI detections}

Inspection of the results listed in Table 2 reveals we have detected interstellar OVI with a significance $>2.8-\sigma$ in only five of the 17 sight-lines sampled (i.e. $~ 30 \%$ ), with no significant OVI absorption being detected along sight-lines with distances $<80 \mathrm{pc}$. The column density values for the five detections range from $\log N(\mathrm{OVI})=12.98$ to $13.48 \mathrm{~cm}^{-2}$, which can be compared with a median value of $13.06 \mathrm{~cm}^{-2}$ found for the 23 (uncontaminated) interstellar OVI detections with $>2-\sigma$ significance observed towards hot white dwarf stars with distances $<200 \mathrm{pc}$ in Table 3 of Savage \& Lehner (2006). Our present value of N(OVI) can also be compared to that of $\log N(\mathrm{OVI})=13.12 \mathrm{~cm}^{-2}$, which is the average value derived from Copernicus satellite observations of 13 B-type stars located within 200 pc that possess OVI detections (Bowen et al. 2008).

Although our 5 OVI detections are along sight-lines with distances $>80 \mathrm{pc}$, we note that along similarly distant path lengths to HD 75311 (168 pc), HD 90972 (147 pc), HD 150100 (123 pc) and HD 188665 (196 pc) we have only measured (2- $\sigma$ ) detection upper limits of $\log N(\mathrm{OVI})<12.93 \mathrm{~cm}^{-2}$. Both Oegerle et al. (2005) and Savage \& Lehner (2006) have found a non-uniform local distribution of OVI, both in terms of the position on the sky and in the distance of the sight-line sampled within $200 \mathrm{pc}$. Thus, our present results would seem to support the prevailing explanation for the spatial distribution of OVI in the local ISM in that it appears in "patches" and it is not part of a local uniformly distributed hot and gaseous medium. We shall return to this point later in Sect. 3.5.

Since the majority of our observations have resulted in nondetections of the OVI absorption-line (due to a combination of the low $\mathrm{S} / \mathrm{N}$ of the FUSE data and the intrinsically weak level of OVI absorption in the local ISM), a more instructive and measurable quantity is the OVI volume density, $n_{\mathrm{OVI}}=N(\mathrm{OVI}) / d$, as listed in Table 2 for the 17 sight-lines. The values of $n_{\text {OVI }}$ for the five detections range from 2.0 to $7.3 \times 10^{-8} \mathrm{~cm}^{-3}$, with an average value of $5.2 \times 10^{-8} \mathrm{~cm}^{-3}$. This average value can be directly compared with that of $n_{\mathrm{OVI}}=4.6 \times 10^{-8} \mathrm{~cm}^{-3}$, determined from the average of the interstellar detections with $\geq 2-\sigma$ significance in the local ISM OVI survey of Savage \& Lehner (2006). An average value of $n_{\mathrm{OVI}}=3.7 \times 10^{-8} \mathrm{~cm}^{-3}$ is obtained from the 
13 detections of OVI from Copernicus satellite observations of B-stars within 200 pc (Bowen et al. 2008).

The average of these three separate determinations of $n_{\mathrm{OVI}}$ is $4.5 \times 10^{-8} \mathrm{~cm}^{-3}$, which agrees with the original results of Shelton \& Cox (1994), who first showed that the LC has a higher than average OVI volume density than that found for more distant galactic regions. We note that a value of $1.3 \times 10^{-8} \mathrm{~cm}^{-3}$ has recently been determined for the mid-plane OVI volume density in the more distant (and higher signal-to-noise) galactic survey of the OVI ion (Bowen et al. 2008). Thus, our data (although of low signal-to-noise and mostly comprised of upper limit values) concurs with previous authors in that there is an over-density of local OVI compared with the general ISM.

Savage \& Lehner (2006) found that the velocity difference, $V_{\mathrm{OVI}}-V_{\mathrm{CII}}$, was always a positive value for the 24 sight-lines which had $\geq 2-\sigma$ OVI and CII interstellar detections. They also found a good correlation between the velocities of interstellar OI and CII. For the 5 sight-lines that have OVI detections in our B-star sample, only three of them have a positive velocity difference. However, given the uncertainty in the wavelength scale assigned by the FUSE data pipeline, we do not assign any significance to this present finding.

\subsection{OVI upper limits}

For our sample of 12 sight-line non-detections of OVI, if we treat these 2- $\sigma$ upper limit values for $n_{\mathrm{OVI}}$ as actual detections we then determine a median upper limit value of $\leq 3.3 \times 10^{-8} \mathrm{~cm}^{-3}$ for the OVI volume density for sight-lines $<200 \mathrm{pc}$ in the local ISM. This value is to be compared with the median value of $n_{\mathrm{OVI}} \leq$ $3.7 \times 10^{-8} \mathrm{~cm}^{-3}$ obtained using the same criterion for the sightline upper limits in the Savage \& Lehner (2006) OVI survey. Similarly, a median value of $n_{\mathrm{OVI}} \leq 3.7 \times 10^{-8} \mathrm{~cm}^{-3}$ is obtained for the 7 non-detections of OVI using Copernicus satellite observations of B-stars within 200pc (Bowen et al. 2008). Thus, our derived estimates of the volumic density of OVI, whether based on detections or upper limits, are in good agreement with previously determined values.

\subsection{Doppler line widths}

Although we have only five OVI detections, we find that the average doppler width (i.e. $b$-value) of the OVI absorption profiles is $b=16.3 \pm 3.0 \mathrm{~km} \mathrm{~s}^{-1}$. This falls just within the range of doppler broadenings $23.0 \pm 5.6 \mathrm{~km} \mathrm{~s}^{-1}$ found by Savage \& Lehner (2006) for the $>4$-sigma detections in their survey. We note that the thermal doppler broadening of OVI near its temperature of peak abundance is $b=17.0 \mathrm{~km} \mathrm{~s}^{-1}$, which corresponds to a temperature of $\sim 2.85 \times 10^{5} \mathrm{~K}$. This temperature is consistent with what one might expect for a hot gas originating in the evaporative outflow from a conductive interface between warm $(T<20000 \mathrm{~K})$ and hot $\left(T \sim 10^{6} \mathrm{~K}\right)$ gas, as predicted by several theoretical models (Slavin \& Frisch 2002; Indebetouw \& Shull 2004).

\subsection{Ol and Cll absorption characteristsics}

The interstellar OI $\lambda 1039 \AA$ and CII $\lambda 10363 \AA$ lines have been well-detected along all of the sight-lines (except for HD 20863 and HD 148594, see Table 2). Both of these absorption lines are near (or at) saturation level and therefore since their column density values cannot be well constrained using these data alone, we have not attempted to fit their profiles. However, we have measured their equivalent widths and central absorption velocities for comparison purposes with other ions. A plot of the central velocities of OI versus CII for the 16 common sight-lines produces a Spearman rank correlation statistic of $r^{2}=0.82$, which supports the findings of Savage \& Lehner (2006) who also found a good correlation between their central absorption velocities.

The OI ion is a good tracer of neutral regions, having a similar ionization potential to that of $\mathrm{HI}$ (i.e. $13.61 \mathrm{eV}$ ), and has an $\mathrm{OI} / \mathrm{HI}$ ratio of $3.45 \times 10^{-4}$, as derived for several sightlines within 100 pc Oliveira et al. (2006). The nominal neutral boundary to the LC has been estimated from NaI absorption observations to many sight-lines within 200 pc by Sfeir et al. (1999), and has been defined as the region in which a rapid build-up, or "wall", of neutral gas is encountered over a short distance. This cold and dense boundary to the inner rarefied LC has an estimated neutral hydrogen column density of $\log N(\mathrm{HI})=19.3-20.0 \mathrm{~cm}^{-2}$, that is contained within a gas "shell" of $\sim 40 \mathrm{pc}$ thickness in most directions. For an OI equivalent width of $>150 \mathrm{m \AA}$ this corresponds to a column density of $N(\mathrm{OI}) \geq 15.6 \mathrm{~cm}^{-2}$, the minimum value which Lehner et al. (2003) has assigned for sight-lines crossing the LC neutral boundary. Based on the OI equivalent widths listed in Table 2, only the sight-line to HD $148594(d=167 \mathrm{pc})$ meets this definition of definitely lying beyond the LC cold and dense neutral boundary. We also note that based on their OI equivalent width values, all of the five B-star sight-lines with OVI detections are placed within the LC.

\subsection{Is there interstellar OVI within the LC?}

Based on the FUSE OVI survey of the galactic plane by Bowen et al. (2008), it is clear that interstellar OVI absorption is commonly encountered along sight-lines extending well-beyond the neutral boundary of the LC. However, is interstellar OVI also to be found as frequently along sight-lines contained within the rarefied LC cavity (i.e. at distances $<80 \mathrm{pc}$ )? Both Oegerle et al. (2005) and Savage \& Lehner (2006) have forwarded several compelling arguments in support of (albeit weak) interstellar OVI absorption arising within the confines of the LC. In their sample of 24 hot white dwarfs located within 200 pc with $\geq 2 \sigma$ OVI detections, there are 5 stars located within 70 pc (i.e. placed within the LC) that have measurable absorption features near the wavelength of OVI. However, given the newly realized complexity of the stellar atmospheres and the circumstellar environments surrounding many hot white dwarfs as outlined in the Introduction to this paper, can these 5 absorption features still be unequivocally associated with an interstellar origin?

Firstly, the 5 OVI detections of Savage \& Lehner (2006) with sight-lines $<70 \mathrm{pc}$ have values of $\log N(\mathrm{OVI})$ ranging from 12.52 to $13.10 \mathrm{~cm}^{-2}$, all detected with significances $>3 \sigma$. Only two of our B-star sight-lines lie unequivocally within the neutral boundary that defines the LC (HD 139006, $d=23 \mathrm{pc}$ and HD $176638, d=56 \mathrm{pc}$ ), and both have $2-\sigma$ detection upper limits of $\log N(\mathrm{OVI})<13.0 \mathrm{~cm}^{-2}$. Thus, given the low $\mathrm{S} / \mathrm{N}$ data of our FUSE B-star data, we would be unable to positively detect the expectedly low absorption levels of locally formed OVI with any degree of confidence for sight-lines $<50 \mathrm{pc}$. However, we are able to claim that we typically find that $\log N(\mathrm{OVI})<$ $13.0 \mathrm{~cm}^{-2}$ for interstellar sight-lines $<70 \mathrm{pc}$. As discussed at length by Savage \& Lehner (2006), current theoretical models predict values of $\log N(\mathrm{OVI}) \sim 12.0$ to $13.3 \mathrm{~cm}^{-2}$ per cloud during both the evaporative and condensation phases of the 

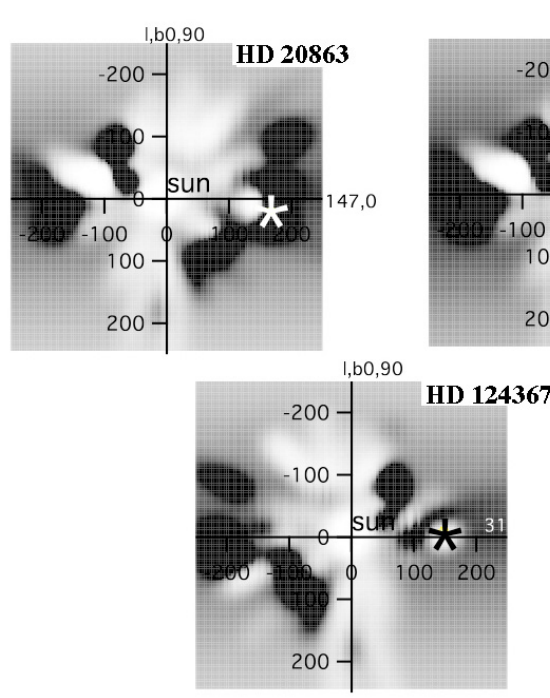
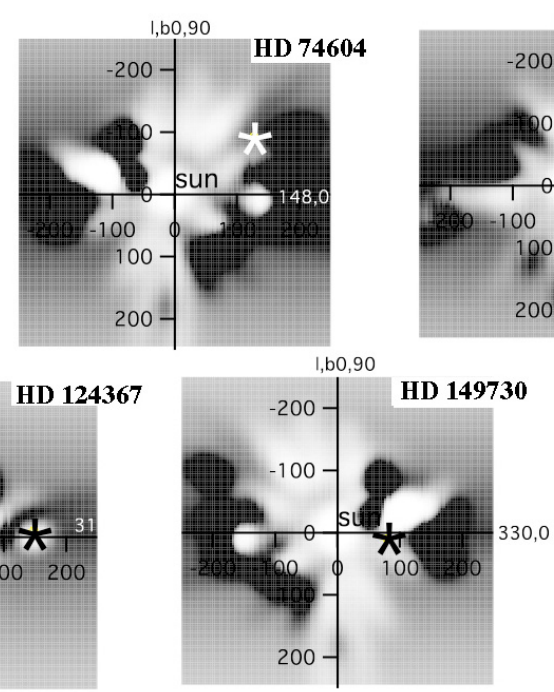

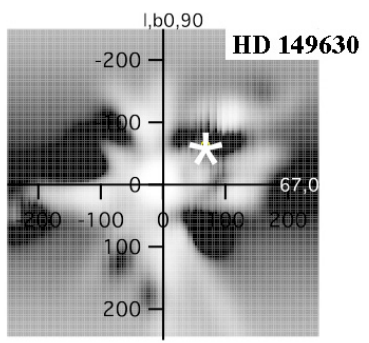

Fig. 4. Location of the 5 B-type stars with detectable OVI absorption with respect to the neutral gas density contours derived by Lallement et al. (2003) for the Local Cavity region. Each of the 5 maps lists the relevant projection (in galactic longitude and latitude) required to produce the gas volume density distribution (in black) to a distance of 200 pc in all directions. White volumes typically have values of $N(\mathrm{NaI})<5 \times 10^{10} \mathrm{~cm}^{-2}$, while black (dense) volumes have an average $\mathrm{NaI}$ density some 20-50 times higher. evolution of an interstellar conductive interface. Thus, our presently observed values of $N(\mathrm{OVI})$ found within $200 \mathrm{pc}$ are fully consistent with current OVI theory.

Finally, for the 5 B-stars for which we have positively detected OVI absorption, in Figs. 4a to e we show their locations with respect to the local density distribution of $\mathrm{NaI}$ absorption (i.e. cold and dense gas) as shown in Lallement et al. (2003). It can be seen that in all 5 cases the B-stars lie just within, or just at, the dense neutral boundary to the LC. It should be noted that there is a typical uncertainty of $\sim 20 \mathrm{pc}$ in the placement of the gas density features in these maps (due to the limited number of sight-lines used in sampling NaI absorption in the local ISM), in addition to the errors in the Hipparcos stellar distances listed in Table 1.

As stated in Sect. 3.4, the OI data towards these 5 stars also suggest that they are not located well beyond the neutral boundary to the LC. Thus, it seems highly likely that the OVI absorption we have presently detected can reasonably be associated with its formation just within the LC. Since the location of the 5 targets for which OVI was detected all lie very close to the neutral boundary of the LC, this may be of significance with regard to the production mechanisms for interstellar OVI. This high ion is widely believed to form in regions in which cool and warm gas $\left(10^{2}-10^{4} \mathrm{~K}\right)$ interface with hot $\left(10^{6} \mathrm{~K}\right)$ gas, resulting in layers containing transition temperature gas at $\sim 300000 \mathrm{~K}$. Thus, if we assume that the neutral boundary to the LC provides the cool interface for the production of OVI, then we must also require a million degree $\mathrm{K}$ gas located within that cavity in order to form the required OVI transition layer.

Of the five sight-lines $<70 \mathrm{pc}$ along which OVI was detected with a significance $>3-\sigma$ by Savage \& Lehner (2006), four are located at galactic latitudes with $+30^{\circ}<b<-30^{\circ}$. An excess of $N(\mathrm{OVI})$ was also reported by these authors for sight-lines towards the north galactic polar region compared with lower latitude directions. We note that this is the galactic region with the highest levels of soft X-ray background emission recorded by the ROSAT (Snowden et al. 1997), and represents directions pointing away from the galactic plane along the axis of the rarefied Local Chimney (Welsh et al. 1999). We also note that McCammon et al. (2002) have made a tentative detection of the Fe IX, X and XI line complex in a region centered at $(l=$ $\left.90^{\circ}, b=+60^{\circ}\right)$, which suggests the presence of million degree gas in this high latitude direction. Similarly, the majority of the sight-lines along which OVI has been detected in emission also occurs at similarly high galactic latitudes of $+30^{\circ}<b<-30^{\circ}$ (Dixon et al. 2006). In addition, both of the Copernicus OVI absorption detections within 80 pc (i.e. $\alpha$ Vir and $\alpha$ Eri) also occur at high galactic latitudes (Bowen et al. 2008). Taken together, all these various observations seem to provide tentative evidence that the spatial distribution of OVI as detected in both emission and absorption may well be loosely correlated with the spatial distribution of the SXRB at these moderately high latitudes for sight-lines lying within the Local Cavity $(d<80 \mathrm{pc})$. The presence of a hot $10^{6} \mathrm{~K}$ emitting gas mainly located at high latitudes but also lying within the LC (presumably lying just above/below the nearby collection of local warm cloudlets) could therefore supply the transition layer required for the formation of OVI absorption. This, admittedly speculative, scenario may also provide an explanation for the recent findings of Lallement (2004) and Koutroumpa et al. (2008). These authors contend that a significant fraction of the SXRB signal in the galactic plane arises within the heliosphere due to charge exchange processes, whereas this production mechanism cannot account for the levels of SXRB emission observed at higher galactic latitudes, which is thought to be associated with an infalling hot halo gas. Beyond the neutral contours of the LC (i.e. $d>80 \mathrm{pc}$ ) the apparent distribution of OVI absorbers (as shown both by our present absorption results and the OVI survey of Bowen et al. 2008) favors a production mechanism located at the interfaces between more distant hot bubble cavities and their surrounding neutral gas shells. Thus, it may be that there are two distinctly different spatial distributions of OVI absorption: (i) one for moderately high galactic latitudes along sight-lines $<80 \mathrm{pc}$; and (ii) one associated with the more distant conduction interfaces of hot interstellar cavities and their surrounding neutral gas shells.

Alternately, we note that recent simulations of the formation of the LC suggest that it is a long-lived ( $14 \mathrm{Myr})$ remnant carved out of the ISM by multiple supernova explosions (Breitschwerdt \& de Avillez 2006; Smith \& Cox 2001). In the latter model, the last re-heating of the LC was thought to be caused by a nearby supernova some 3 million years ago, with evidence for such a scenario being based on the excess abundance of ${ }^{60} \mathrm{Fe}$ found in the deep ocean crust (Fields et al. 2005). 
The evolution of a $S N R$ located within an existing low-density interstellar environment has been modeled for emission from both high ion stages (Shelton 1998) and soft X-rays (Shelton 1999). Her models show that a middle-aged SNR would have hot gas in the interior and zone of OVI-rich gas at the boundary, and as time elapses the hot gas in the interior cools sufficiently for its X-ray emission to fade significantly. In particular, the model predicts that within a time-scale of $\sim 2.5 \times 10^{5} \mathrm{yr}$ the high-stage ions (such as OVI and NV) are mainly found at the periphery of the hot SNR bubble and the rapidly cooling central gas is overionized and out of equilibrium (Shelton 1998). By $10^{6} \mathrm{yr}$ the central regions have cooled sufficiently that the level of X-ray emission is negligible, with appreciable amounts of OVI ions lying within warm gas at the bubble's edge. This type of model could go a long way in explaining both the location of OVI absorption presently observed at the neutral boundary to LC and the far low levels of SXRB emission intensity found within the galactic plane as required with models of solar wind charge exchange emission by Koutroumpa et al. (2008).

\section{Conclusion}

We have presented far ultraviolet (FUV: 990-1082 Å) spectroscopic observations of 17 early-type stars located within $200 \mathrm{pc}$ of the Sun in order to measure interstellar absorption due to the important OVI (1032 ̊) ion. Only 5 significant detections of OVI have been made, all towards stars with distances $>80 \mathrm{pc}$ and all with values of $\log N(\mathrm{OVI}) \geq 12.98 \mathrm{~cm}^{-2}$. The average volumic density, $n_{\mathrm{OVI}}$, along these 5 sight-lines is $5.2 \times 10^{-8} \mathrm{~cm}^{-3}$, which is measurably higher than that found throughout the general ISM. Along the 12 sight-lines for which no interstellar OVI was detected, we have determined a median value for $n_{\mathrm{OVI}}<$ $3.3 \times 10^{-8} \mathrm{~cm}^{-3}$. Both of these values for $n_{\mathrm{OVI}}$ are consistent with previous measurements made towards hot white dwarf stars located within 200 pc by Oegerle et al. (2005) and Savage \& Lehner (2006).

The 5 stars with detectable OVI are all located just within, or just at, the neutral boundary to the LC. Their placement at this boundary suggests that the OVI is formed in a conductive interface within a transition region between hot million degree gas within the LC and cool neutral gas at the LC boundary. We also provide tentative evidence that the spatial distribution of very local $(d<80 \mathrm{pc})$ OVI absorbers and emitters exhibits a loose correlation with that of the million degree $\mathrm{K}$ soft $\mathrm{X}$-ray background emission at galactic latitudes with $+30^{\circ}<b<-30^{\circ}$. In such a scenario very little SXRB emission is required in the galactic plane, in agreement with current theoretical models that invoke solar wind charge exchange as the origin for the majority of this emission. Alternately, it may be that the OVI is the current byproduct of an old $\mathrm{SN}$ that exploded within the LC $\sim 3$ million years ago and the central regions of the LC may no longer contain significant amounts of soft X-ray emitting gas,

Acknowledgements. This publication makes use of data products from the SIMBAD database, operated at CDS, Strasbourg, France. B.Y.W. would like to acknowledge funding for this research through NASA grant \# NNX07AE33G.

\section{References}

Andersson, B.-G., \& Potter, S., 2006, ApJ, 640, L51

Andre, M., Oliveira, C., Howk, J., et al. 2003, ApJ, 591, 1000

Bannister, N., Barstow, M. A., Holberg, J., \& Bruhweiler, F. 2003, MNRAS, 341, 477

Barstow, M. A., Boyce, D., Barstow, J., et al. 2008, Astr. Sp. Sci., tmp, 119B (on-line)

Bjorkman, J., Ignace, R., Tripp, T., \& Cassinelli, J. 1994, ApJ, 435, 416

Bloch, J., Jahoda, K., Juda, M., et al. 1986, ApJ, 308, L59

Bowen, D., Jenkins, E., Tripp, T., et al. 2008, ApJS, 176, 59

Breitschwerdt, D., \& de Avillez, M. 2006, A\&A, 452, L1

Cox, D., \& Smith, R. 1974, ApJ, 189, L105

Cox, D., \& Helenius, L. 2003, ApJ, 583, 205

Crawford, I. 2000, MNRAS, 317, 996

Dixon, W. V., Sankritt, R., \& Otte, B. 2006, ApJ, 647, 328

Dixon, W. V., Sahnow, D., Barrett, P., et al. 2007, PASP, 119, 527

ESA 1997, The Hipparcos and Tycho catalogues, ESA SP-1200

Fields, B., Hochmuth, K., \& Ellis, J. 2005, ApJ, 621, 902

Henley, D., \& Shelton, R. 2007, BAAS, 210, 1912

Holberg, J., Barstow, M., Lanz, T., \& Hubeny, I. 1997, ApJ, 484, 871

Holberg, J., Barstow, M., \& Sion, E. 1998, ApJS, 119, 207

Hurwitz, M., Sasseen, T., \& Sirk, M. 2005, ApJ, 623, 911

Indebetouw, R., \& Shull, M. 2004, ApJ, 605, 205

Jelinsky, P., Vallerga, J. V., \& Edelstein, J. 1995, ApJ, 442, 653

Jenkins, E. B. 1978, ApJ, 219, 845

Jenkins, E. 2002, ApJ, 580, 938

Kilic, M., \& Redfield, S. 2007, ApJ, 660, 641

Koester, D., \& Wilken, D. 2006, A\&A, 453, 1051

Koutroumpa, D., Acero, F., Lallement, R., Ballet, J., \& Kharchenko, V. 2007, A\&A, 475, 901

Koutroumpa, D., Lallement, R., Kharchenko, V., \& Dalgarno, A. 2008, Sp. Sci. Rev., in press

Lallement, R. 1998, The Local Bubble and Beyond, IAU Colloq., 506, 19

Lallement, R. 2004, A\&A, 418, 143

Lallement, R., Vidal-Madjar, A., \& Ferlet, R. 1986, A\&A, 168, 225

Lallement, R., Welsh, B. Y., Vergely, J. L., Crifo, F., \& Sfeir, D. 2003, A\&A, 411,447

Lehner, N., Jenkins, E., Gry, C., et al. 2003, ApJ, 595, 858

McCammon, D., Almy, R., Apodaca, E., et al. 2002, ApJ, 576, 188

McKee, C., \& Ostriker, P. 1977, ApJ, 218, 148

Moos, H. W., Cash, W. C., Cowie, L. L., et al. 2000, ApJ, 538, L1

Moos, H. W., Sembach, K. R., Vidal-Madjar, A., et al. 2002, ApJS, 140, 3

Oegerle, W. R., Jenkins, E. B., Shelton, R. L., Bowen, D. V., \& Chayer, P. 2005, ApJ, 622, 377

Oliveira, C., Dupuis, J., Chayer, O., \& Moos, H. W. 2005, ApJ, 625, 232

Robertson, I., \& Cravens, T. 2003, JGR, 108, 8031

Sahnow, D., Moos, H. W., Ake, T., et al. 2000, ApJ, 538, L7

Sanders, W., Kraushaar, W., Nousek, J., \& Fried, P. 1977, ApJ, 217, L87

Sanders, W. T., Edgar, R. J., Kraushaar, W. L., McCammon, D., \& Morgenthaler, J. P. 2001, ApJ, 554, 694

Savage, B., \& Lehner, N. 2006, ApJS, 162, 134

Sfeir, D., Lallement, R., Crifo, F., \& Welsh, B. Y. 1999, A\&A, 346, 785

Shelton, R. 1998, ApJ, 504, 785

Shelton, R. 1999, ApJ, 521, 217

Shelton, R., \& Cox, D. 1994, ApJ, 434, 599

Slavin, J., \& Frisch, P. 2002, ApJ, 565, 364

Smith, R. K., \& Cox, D. 2001, ApJS, 134, 283

Smith, R. K., Bautz, M. W., Edgar, R. J., et al. 2007, PASJ, 59, 141

Snowden, S., Egger, R., Freyberg, M., et al. 1997, ApJ, 485, 125

Snowden, S. L., Egger, R., Finkbeiner, D. P., Freyberg, M. J., \& Plucinsky, P. P. 1998, ApJ, 493, 715

Watson, J., \& Meyer, D. 1996, ApJ, 473, L127

Welsh, B. Y., \& Lallement, R. 2008, A\&A, in preparation

Welsh, B. Y., Vedder, P., \& Vallerga, J. 1990, ApJ, 358, 473

Welsh, B. Y., Sfeir, D., Sirk, M., \& Lallement, R. 1999, A\&A, 352, 308

Williamson, F. O., Sanders, W. T., Kraushaar, W. L., et al. 1974, ApJ, 193, L133

Zuckerman, B., Koester, D., Reid, I., \& Hunsch, M. 2003, ApJ, 596, 477 